\title{
A COMPARISON THEOREM FOR ELLIPTIC EQUATIONS ${ }^{1}$
}

M. H. PROTTER

Hartman and Wintner [1] obtained a Sturmian comparison theorem for self-adjoint second order linear elliptic equations of the form

$$
\sum_{i, j=1}^{n} \frac{\partial}{\partial x_{i}}\left(a_{i j} \frac{\partial u}{\partial x_{j}}\right)+f u=0, \quad a_{i j}=a_{j i}
$$

in a bounded domain $D$ with boundary $\Gamma$. It is the purpose of this note to extend their result to general second order linear elliptic equations. Let $v$ be a solution of another elliptic equation of the same form

$$
\sum_{i, j=1}^{n} \frac{\partial}{\partial x_{i}}\left(\alpha_{i j} \frac{\partial v}{\partial x_{j}}\right)+F v=0, \quad \alpha_{i j}=\alpha_{j i}
$$

and denote the elements of the inverse matrices by $a^{i j}$ and $\alpha^{i i}$ respectively.

Let $u(x)=u\left(x_{1}, \cdots, x_{n}\right)$ be a solution of (1) which is nonnegative in $D$ and vanishes on $\Gamma$. We exclude the trivial case $u \equiv 0$. Suppose that the coefficients of (1) and (2) satisfy the relations

$$
\begin{gathered}
\left\|\alpha^{i j}-a^{i j}\right\| \text { is non-negative definite in } D, \\
f \leqq F \text { in } D .
\end{gathered}
$$

The theorem of Hartman and Wintner states that if (3) and (4) hold then any solution of (2) must have a zero in $D+\Gamma$.

We now consider in $D$ two general linear elliptic equations of the form

$$
\begin{array}{ll}
\sum_{i, j=1}^{n} \frac{\partial}{\partial x_{i}}\left(a_{i j} \frac{\partial u}{\partial x_{j}}\right)+\sum_{i=1}^{n} b_{i} \frac{\partial u}{\partial x_{i}}+f u=0, & a_{i j}=a_{j i}, \\
\sum_{i, j=1}^{n} \frac{\partial}{\partial x_{i}}\left(\alpha_{i j} \frac{\partial v}{\partial x_{j}}\right)+\sum_{i=1}^{n} \beta_{i} \frac{\partial v}{\partial x_{i}}+F v=0, & \alpha_{i j}=\alpha_{j i}
\end{array}
$$

and determine conditions on the coefficients so that a Sturmian comparison theorem shall be valid. For simplicity we consider the case of two independent variables, the result in more than two vari-

Received by the editors July 26, 1958.

1 This research was supported by the United States Air Force through the Air Force Office of Scientific Research of the Air Research and Development Command under Contract No. AF 49(638)-398. 
ables being a straightforward extension. That is, we consider the equations

$$
\begin{gathered}
L_{1} u \equiv\left(a u_{x}\right)_{x}+\left(b u_{x}\right)_{y}+\left(b u_{y}\right)_{x}+\left(c u_{y}\right)_{y} \\
+2 d u_{x}+2 e u_{y}+f u=0 \\
L_{2} v \equiv\left(\alpha v_{x}\right)_{x}+\left(\beta v_{x}\right)_{y}+\left(\beta v_{y}\right)_{x}+\left(\gamma v_{y}\right)_{y} \\
+2 \delta v_{x}+2 \epsilon v_{y}+F v=0 .
\end{gathered}
$$

An application of Green's theorem to (7) for a function $u$ which vanishes on $\Gamma$ yields

$-\iint_{D} u L_{1} u d x d y$

$$
=\iint_{D}\left[a u_{x}^{2}+2 b u_{x} u_{y}+c u_{y}^{2}-2 d u u_{x}-2 e u u_{y}-f u^{2}\right] d x d y .
$$

We now employ a device of Picard [3] extended by Hartman and Wintner [1]. The same technique was also used by Ou and Ding [2] and the author in connection with certain problems in equations of mixed type. Let $P$ and $Q$ be arbitrary smooth functions in $D$. Then for any function $u$ vanishing on $\Gamma$ we have

$$
\iint_{D}\left[\frac{\partial}{\partial x}\left(P u^{2}\right)+\frac{\partial}{\partial y}\left(Q u^{2}\right)\right] d x d y=0 .
$$

Thus if in addition $u$ is a solution of (7) we find

$$
\begin{aligned}
\iint_{D}\left[a u_{x}^{2}\right. & +2 b u_{x} u_{y}+c u_{y}^{2}+2(P-d) u u_{x} \\
& \left.+2(Q-e) u u_{y}+\left(P_{x}+Q_{y}-f\right) u^{2}\right] d x d y=0 .
\end{aligned}
$$

The condition that the integrand be a positive semi-definite form in $u, u_{x}, u_{y}$ is

$$
\begin{aligned}
\left(a c-b^{2}\right)\left(P_{x}+Q_{\nu}-f\right) & \\
& \geqq a(Q-e)^{2}-2 b(P-d)(Q-e)+c(P-d)^{2} .
\end{aligned}
$$

Define

$$
P=\frac{-\alpha v_{x}-\beta v_{y}}{v}+d-\xi, \quad Q=\frac{-\beta v_{x}-\gamma v_{y}}{v}+e-\eta
$$

where $v$ is a positive solution of (8) and $\xi, \eta$ are functions yet to be determined. Substitution for $P$ and $Q$ in (10) yields the expression 


$$
\begin{aligned}
&\left(a c-b^{2}\right)\left(\alpha v_{x}^{2}+2 \beta v_{x} v_{y}+\gamma v_{y}^{2}\right) \\
& \quad+\left(a c-b^{2}\right) v\left[2 \delta v_{x}+2 \epsilon v_{y}+\left(F-f-\xi_{x}-\eta_{y}+d_{x}+e_{y}\right) v\right] \\
& \geqq\left(a \beta^{2}-2 b \alpha \beta+c \alpha^{2}\right) v_{x}^{2}+2\left(a \beta \gamma-b \beta^{2}-b \alpha \gamma+c \alpha \beta\right) v_{x} v_{y} \\
&+\left(a \gamma^{2}-2 b \beta \gamma+c \beta^{2}\right) v_{y}^{2}+2(a \beta \eta+c \alpha \xi-b \alpha \eta-b \beta \xi) v v_{x} \\
&+2(a \eta \gamma+c \beta \xi-b \beta \eta-b \gamma \xi) v v_{y} \\
&+\left(a \eta^{2}-2 b \xi \eta+c \xi^{2}\right) v^{2} .
\end{aligned}
$$

We now investigate conditions on the coefficients of (7) and (8) which will make the above inequality hold throughout $D$. This will contradict (9) and hence $v$ must have a zero in $D$. If condition (3) is satisfied, which in this case becomes

$$
a>\alpha, \quad(a-\alpha)(c-\gamma)-(b-\beta)^{2} \geqq 0,
$$

then (11) will be a consequence of the inequality

$$
\begin{aligned}
\left(a c-b^{2}\right) v\left[2 \delta v_{x}\right. & \left.+2 \epsilon v_{y}+\left(F-f-\xi_{x}-\eta_{y}+d_{x}+e_{y}\right) v\right] \\
\geqq & 2(a \beta \eta+c \alpha \xi-b \alpha \eta-b \beta \xi) v v_{x} \\
& +2(a \eta \gamma+c \beta \xi-b \beta \eta-b \gamma \xi) v v_{y}+\left(a \eta^{2}-2 b \xi \eta+c \xi^{2}\right) v^{2} .
\end{aligned}
$$

We select $\xi, \eta$ to be solutions of the linear equations

$$
\begin{aligned}
& (a \beta-b \alpha) \eta+(c \alpha-b \beta) \xi=-\delta\left(a c-b^{2}\right), \\
& (a \gamma-b \beta) \eta+(c \beta-b \gamma) \xi=-\epsilon\left(a c-b^{2}\right) .
\end{aligned}
$$

That this is always possible follows from the ellipticity of the operators $L_{1}$ and $L_{2}$. Hence (11) is now a consequence of

$$
\left(a c-b^{2}\right)\left(F-f-\xi_{x}-\eta_{y}+d_{x}+e_{y}\right)-\left(a \eta^{2}-2 b \xi \eta+c \xi^{2}\right) \geqq 0 .
$$

We therefore have the result: if the coefficients of (7) and (8) satisfy relations (12) and (13) and if there is a non-negative solution of (7), not identically zero, which vanishes on $\Gamma$, then evcry solution of (8) must have a zero in $D+\Gamma$.

In the special case $\delta=\epsilon \equiv 0$ we have $\xi=\eta \equiv 0$ and condition (13) becomes

$$
F-f+d_{x}+e_{y} \geqq 0 .
$$

This is particularly useful if a comparison is desired between a general linear second order equation and the equation

$$
\Delta u+m u=0
$$

$m$ a constant. Then we have the conditions 


$$
\begin{gathered}
a>1, \quad(a-1)(c-1)-b^{2} \geqq 0, \\
m \geqq-d_{x}-e_{y}+f .
\end{gathered}
$$

Of course a more general relation between the coefficients, sufficient to yield the conclusion of the theorem, can be obtained by imposing the condition that (11) be a positive semi-definite form in $v, v_{x}, v_{y}$.

\section{BIBLIOGRAPHY}

1. P. Hartman and A. Wintner, On a comparison theorem for self-adjoint partial differential equations of elliptic type, Proc. Amer. Math. Soc. vol. 6 (1955) p. 862.

2. Sing-Mo Ou and Shia-Shi Ding, Sur l'unicitê du problème de Tricomi de l'équation de Chaplygin, Acta Math. Sinica vol. 5 (1955) p. 393.

3. E. Picard, Mémoire sur la théorie des équations aux dérivées partielles et la méthode des approximations successives, J. Math. Pures Appl. vol. 6 (1890) p. $145 \mathrm{ff}$.

University of CALIfornia, Berkeley 